\title{
Prognostic factors of mid-term clinical outcome in congestive heart failure patients discharged after acute decompensation
}

\author{
Mauro Feola ${ }^{1}$, Enrico Lombardo ${ }^{1}$, Marzia Testa², Enrico Avogadri ${ }^{1}$, Salvatore Piccolo ${ }^{1}$, Antonello Vado ${ }^{3}$
}

1Cardiovascular Rehabilitation-Heart Failure Unit, Ospedale SS Trinita', Fossano, Italy
2Geriatrics and Bone Diseases, University of Turin, Italy

${ }^{3}$ Department of Cardiovascular Diseases, Ospedale Santa Croce-Carle Cuneo, Italy

Submitted: 17 March 2011

Accepted: 5 December 2011

Arch Med Sci 2012; 8, 3: 462-470

DOI: 10.5114 /aoms.2012.29401

Copyright (c) 2012 Termedia \& Banach

\begin{abstract}
Introduction: Risk stratification in congestive heart failure (CHF) patients is based on a variety of clinical and laboratory variables. We analysed renal function, BNP, water composition, echocardiographic and functional determinations in predicting mid-term outcome in CHF patients discharged after decompensation.

Material and methods: All subjects with NYHA class II-IV were enrolled at hospital discharge. NYHA class, BNP, water body composition, non-invasive cardiac output and echocardiogram were analysed. Death, cardiac transplantation and hospital readmission for CHF were scheduled.

Results: Two-hundred and thirty-seven $(64.5 \%$ males, age $71.1 \pm 10.1)$ patients were discharged after obtaining normal hydration; left ventricular ejection fraction (LVEF) was $43.2 \pm 16.2 \%$, cardiac output was $3.8 \pm 1.1 \mathrm{l} / \mathrm{min}$ and BNP at discharge resulted $401.3 \pm 501.7 \mathrm{pg} / \mathrm{ml}$. During the 14-month follow-up 15 patients (6.3\%) died, 1 (0.4\%) underwent cardiac transplantation and 18 (7.6\%) were readmitted for CHF (event group); in 203 (85.6\%) no events were observed (noevent group). Higher NYHA class ( $2.1 \pm 0.7$ vs. $1.9 \pm 0.4, p=0.01)$, BNP at discharge $(750.2 \pm 527.3 \mathrm{pg} / \mathrm{ml}$ vs. $340.7 \pm 474.3 \mathrm{pg} / \mathrm{ml}, p=0.002)$ and impaired LVEF (33.7 $\pm 15.7 \%$ vs. $44.5 \pm 15.8 \%, p=0.0001)$ and creatinine ( $1.7 \pm 0.6$ vs. $1.2 \pm 0.8 \mathrm{mg} / \mathrm{dl}$, $p=0.004)$ were noticed in the event group. At multivariate Cox analysis LVEF $(p=0.0009)$, plasma creatinine $(p=0.006)$ and BNP at discharge $(p=0.001)$ were associated with adverse mid-term outcome. Kaplan-Meier survival curves demonstrated that adding cut-off points for creatinine $1.5 \mathrm{mg} / \mathrm{dl}$ and discharged BNP of $250 \mathrm{pg} / \mathrm{ml}$ discriminated significantly prognosis ( $p=0.0001$; log rank 21.09).

Conclusions: In predicting mid-term clinical prognosis in CHF patients discharged after acute decompensation, BNP at discharge $\geq 250 \mathrm{pg} / \mathrm{ml}$ added with plasma creatinine $>1.5 \mathrm{mg} / \mathrm{dl}$ are strong adverse predictors.
\end{abstract}

Key words: congestive heart failure, prognosis, natriuretic peptide.

\section{Introduction}

Risk stratification in patients suffering from chronic congestive heart failure (CHF) is based on a variety of clinical and laboratory variables. Indeed, several prognostic parameters have been identified, including age, New York Heart Association (NYHA) class, renal function, comorbidity such as atrial fibrillation, diabetes mellitus and ischaemic heart disease [1]. In acute decompensated heart failure (ADHF) episodes, the degree of renal dysfunction and arterial hypotension easily stratified patients with the

\author{
Corresponding author: \\ Mauro Feola MD, FESC \\ Department of Cardiovascular \\ Rehabilitation-Heart \\ Failure Unit \\ Ospedale SS Trinita' \\ Via Ospedale 4 \\ 12045 Fossano (CN), Italy \\ E-mail: m_feola@virgilio.it
}


worst clinical outcome [2]. A single determination of brain natriuretic peptide (BNP) plasma level represents a reliable risk stratification procedure and its increase is considered a sensitive diagnostic marker of left ventricular dysfunction $[3,4]$. Plasma BNP has clear prognostic relevance in predicting cardiovascular events in congestive heart failure (CHF) patients [5-8] and even in asymptomatic subjects [9].

The objective of this study was to analyse different prognostic parameters (renal function, plasma BNP, water composition, echocardiographic and functional capacity) in predicting mid-term clinical outcome in CHF patients discharged after an ADHF episode.

\section{Material and methods}

\section{Patients}

This prospective cohort study, approved by the local ethics committees, included patients admitted into the Heart Failure Unit from April 2008 to August 2010. All CHF subjects discharged after an acute episode of cardiac decompensation were enrolled in an out-patient clinic follow-up. Patients were classified as having CHF according to the criteria commonly accepted in the literature [10], namely the presence of 2 major criteria or 1 major criterion +2 minor criteria according to the Framingham score and NYHA functional class II, III, or IV, due to exacerbation of symptoms with at least 1 class deterioration. The presence of inadequate echo images or no adherence to the therapy and disagreement with the periodical follow-up were considered exclusion criteria. All patients underwent a clinical examination, a 12-lead electrocardiogram, plasma determination of BNP, water composition (on admission and at discharge), 6-minute walk test (6MWT), non-invasive cardiac output and a transthoracic echocardiogram within $48 \mathrm{~h}$ upon hospital discharge. The criteria for discharging $\mathrm{CHF}$ patients were the following: a) subjective improvement on the basis of NYHA class, with no orthopnoea; b) 90 < systolic blood pressure < $120 \mathrm{~mm} \mathrm{Hg}$; c) heart rate $<100 \mathrm{bpm}$; d) pulse oximetry in ambient air > 90\%; e) diuresis > $1000 \mathrm{ml} /$ day [11] + obtaining normal hydration evaluated with bioelectrical impedance vectorial analysis (see below). Serum creatinine was checked on clinical stability. According to the study protocol, CHF out-patients were checked at 3 and 6 months after discharge. In case of worsening of the clinical status (worsening dyspnoea, body weight increase or oedema, cardiac arrhythmias), a clinical control was provided. The therapy prescribed in those patients included angiotensin-converting enzyme inhibitors (enalapril, ramipril), angiotensin receptor blockade (candesartan, losartan) in case of enalapril/ramipril intolerance, $\beta$-blockers (metoprolol, bisoprolol or carvedilol), digoxin, loop diuretic and spironolactone at low dose. For $\beta$-blockers, angiotensin-converting enzyme inhibitors and angiotensin receptor blockade, the patients' maximum tolerated dose was used, after an adequate titration period.

\section{Doppler echocardiography}

Echocardiograms were performed with a Vivid 7 computed sonography system (GE Medical Systems, Waukesha, Wisconsin, USA) according to the recommendations of the American Society of Echocardiography [12]. Two-dimensional apical 2- and 4-chamber views were used for volume measurements; LVEF was calculated with a modified Simpson's method using biplane apical (2- and 4-chamber) views. The left ventricular (LV) end-diastolic volume and the LV end-systolic volume were recorded. All the echo examinations were performed by expert operators blinded to the results of BNP assay; the intra-observer variability in the evaluation of left ventricular ejection fraction (LVEF) was found to be $<5 \%$. Echocardiographic measurements including LV end-diastolic diameter, and the diastolic thickness of the ventricular septum and the posterior LV wall were determined according to the American Society Echocardiography recommendations [12]. Systolic dysfunction was defined as a level of LVEF $<50 \%$. The definition of restrictive filling pattern (grade 3 ) was a predefined modification of classifications used in prior studies [13]: E/A $\geq 2$, DT $\leq 150 \mathrm{~ms}, \mathrm{~S} / \mathrm{D}$ ratio $<1$, and $A R>35 \mathrm{~cm} / \mathrm{s}$. All these criteria should be verified to define the restrictive filling pattern. The other diastolic filling patterns were classified as: grade 1 (abnormal relaxation) when E/A < 1 with DT $>240$ ms; grade 2 (pseudonormal) when E/A was $0.75-1.5$, DT was 160-240 ms and E/Ea > 15 [13]. The Doppler sample was set $1-2 \mathrm{~mm}$ under the free edges of the mitral valve using the apical 4-chamber projection; an average of 5 beats was considered. In patients suffering from atrial fibrillation at the time of the echocardiogram, the diastolic function was classified as: 1) restrictive pattern (DT $\leq 150 \mathrm{~ms}$ ) or 2) indeterminate (DT > $150 \mathrm{~ms}$ ). All these criteria should be verified to define the restrictive filling pattern: $\leq 150 \mathrm{~ms}, \mathrm{~S} / \mathrm{D}$ ratio $<1$, and $\mathrm{AR}>35 \mathrm{~cm} / \mathrm{s}$. The presence of this diastolic pattern with LVEF $\geq 50 \%$ was defined as an isolated diastolic dysfunction.

\section{BNP assay}

All blood samples were collected by venopuncture and immediately analysed with the bedside Triage $B$ type natriuretic fluorescence immunoassay (Biosite Diagnostics, La Jolla, CA, USA). The Triage Meter is used to measure BNP concentration by detecting a fluorescent emission that reproduces the amount of BNP in the blood. Two hundred and 
fifty microlitres of whole blood was added to the disposable device, then the cells were filtered and separated from the plasma with BNP, which entered a reaction chamber containing fluorescent BNP antibodies. After 2-min incubation, the BNPantibody mixture migrated to an area containing immobilised antibodies and remained fixed. The unbound fluorescent antibodies were washed away by the excess sample fluid. Then, the Triage Meter measured the fluorescent intensity of the BNP assay area. The assay results were complete in 15 min. Performance characteristics of the test: assay range $5-5000 \mathrm{pg} / \mathrm{ml}$; total CV 9.2-11.4\%.

\section{Non-invasive cardiac output}

For the measurement of non-invasive cardiac output (CO), an inert gas rebreathing method (Innocor, Innovison A/S, Odense, Denmark) was used. The system utilised $\mathrm{N}_{2} \mathrm{O}$ (blood soluble gas) and SF6 (blood insoluble gas) enriched with $\mathrm{O}_{2}$ of $0.5 \%$ and $0.1 \%$ respectively. Tidal volume was progressively increased in the closed circuit to match the physiological increase. Use of SF6 allowed measurement of the volume of the lungs, valve and rebreathing bag. $\mathrm{N}_{2} \mathrm{O}$ concentration decreases during the rebreathing manoeuvre, with a rate proportional to pulmonary blood flow. Three to four respiratory cycles were needed to obtain $\mathrm{N}_{2} \mathrm{O}$ washout. Absence of pulmonary shunt was defined as arterial $\mathrm{O}_{2}$ saturation $>98 \%$ (blood sample obtained from the arterial line). In the absence of pulmonary shunt, pulmonary blood flow $=\mathrm{CO}$. This method was proved to be closely correlated with thermodilution $(R=0.93)$ and the direct Fick method $(R=0.94)$ [14].

\section{Six-minute walk test}

The 6MWT was performed on the day of discharge according to the guidelines of the American Thoracic Society [15]. The CHF patients able to walk underwent 6MWT if they did not meet the exclusion criteria (unstable angina and myocardial infarction during the previous month, resting heart rate $>120$; systolic blood pressure $>180 \mathrm{~mm} \mathrm{Hg}$ or diastolic blood pressure $>100 \mathrm{~mm} \mathrm{Hg}$ ). Repeat testing was performed after an adequate rest period in order to minimize the intraday variability.

\section{Bioelectrical impedance vectorial analysis (BIVA)}

Assessment of body fluid status was carried out using an electrical impedance analyser and BodyGram 2.1 software (Akern Pontassieve, Florence Italy). The bioelectrical parameters of resistance, reactance and phase angle were obtained with an electric alternating current flux of $800 \mu \mathrm{A}$ and an operating frequency of $50 \mathrm{kHz}$. Whole body impedance measurements were taken by using a standard position of outer and inner electrodes on the right hand and foot. The entire procedure was performed according to the indications of the National Institutes of Health Technology assessment conference statements [16]. Bioelectrical impedance evaluates some basic properties of the body by measuring resistance, reactance (a form of opposition that electronic components exhibit to the passage of the alternating current counterpart of direct current and which indicates an absolute amount of body cell mass). Bioelectrical impedance is normally used to estimate the volumes of body fluid com-
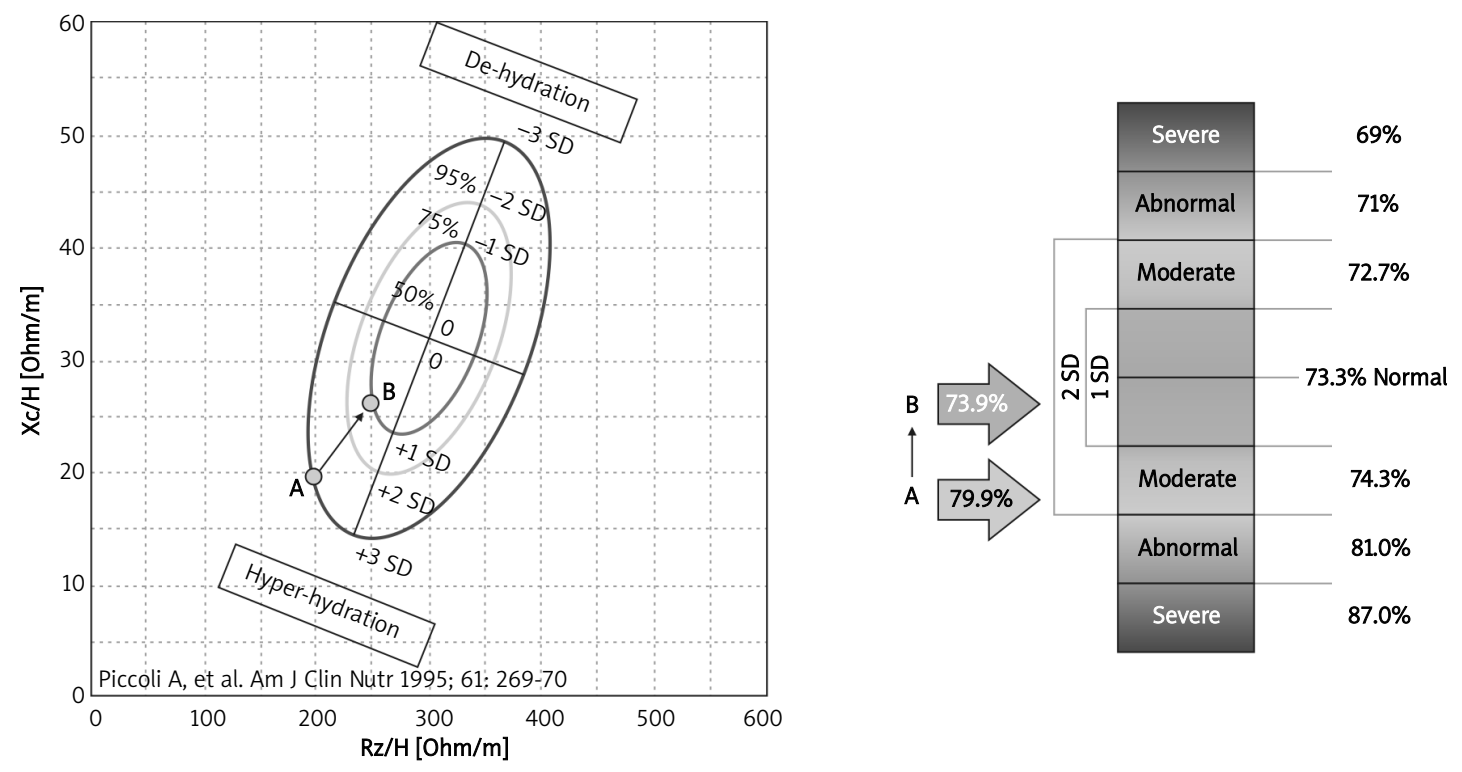

Figure 1. Evaluation of grade of hydration in CHF patients using the BIVA giagram and nomogram; on the left the same patients at time A presents hyper-hydration (+3 SD) that was reduced after diuretic therapy (point B) 
partments allowing one to determine total body water and the ratio between extracellular and total body water. Resistance and reactance were always corrected for the patients' height. The clinical support of this method has been implemented using vector analysis (Bioelectrical Impedance Vector Analysis, Biavector) [17]. The backward or forward position of parallel vectors to the major axis of ellipses is normally correlated with dehydration or hyperhydration (Figure 1). The normal value of hydration was set at a value $73.3 \%$ (nomogram in Figure 1) and the area within +1 SD was considered a satisfactory criterion for discharging CHF patients.

\section{Clinical follow-up}

Death by any cause, cardiac transplantation and worsening heart failure requiring readmission to the hospital were considered cardiovascular events. Data regarding the occurrence of cardiovascular events were collected from multiple sources in all patients.

\section{Statistical analysis}

Continuous variables were expressed as mean \pm standard deviation (SD). We used the Mann-Whitney U-test and the Wilcoxon test for the comparisons between samples, while the association between variables was verified with Fisher's exact test. A $p$ value $<0.05$ was considered significant. Categorical variables (NYHA class, cardiovascular events) were analysed using the $\chi^{2}$ test or Fisher's exact test. The Cox proportional hazard regression model was used to examine the relationship of clinical variables, BNP levels, and echocardiographic parameters with the incidence of the combined endpoint during the 6 months after discharge (hazard ratio and 95\% confidence interval, $\mathrm{Cl}$ ). The hazard ratio for a continuous variable refers to the risk ratio per unit of the considered variable. The BNP levels were evaluated both as a continuous variable and as a categorical variable (based on cut-off values). According to the plasma BNP value, 2 groups were created, $<250 \mathrm{pg} / \mathrm{ml}$ and $\geq 250 \mathrm{pg} / \mathrm{ml}$, in order to evaluate the role of different levels of plasma BNP on the occurrence of cardiac events in the follow-up. Survival curves were estimated according to Kaplan-Meier analysis and compared by the Tarone-Ware test. Analyses were performed using SPSS software for Windows, release 11.0, SPSS Inc., Chicago, USA.

\section{Results}

Two-hundred and thirty-seven patients with a diagnosis of $\mathrm{CHF}$ after an acute decompensation episode entered this cohort study. The mean age was $71.1 \pm 10.1$ years old (range: $30-89$ years old); $64.1 \%$ were males (Table I). The main cause of CHF was ischaemic heart disease (42\%) followed by hypertensive and dilatative cardiomyopathy (Table I). In 12-lead electrocardiogram, 31\% of patients were in permanent atrial fibrillation. On discharge the mean New York Heart Association functional class (NYHA) was $1.9 \pm 0.5$ and the mean plasma BNP was $401.3 \pm 501.7 \mathrm{pg} / \mathrm{ml}$. Renal function was generally impaired (serum creatinine $1.3 \pm 0.8 \mathrm{mg} / \mathrm{dl}$ ).

The transthoracic echocardiogram was performed on all subjects before discharge and a restrictive filling pattern was seen in $22 \%$ of participants, a pseudonormal pattern in $23 \%$ and an impaired relaxation in the majority (55\%) of patients. The mean LVEF was $43.2 \pm 16.2 \%$. Mild mitral regurgitation was found in 115 patients (48\%), while $40(16.8 \%)$ had moderate mitral regurgitation and 21 (8.9\%) severe. The echocardiogram revealed mild or moderate aortic regurgitation in 15 patients (6.3\%). Mild pericardial effusion was found in 18 subjects (7.5\%), without any signs of haemodynamic significance. All the other clinical, biochemical and functional parameters are described in Table I.

The whole population was divided into an events group (34 [14.3\%] subjects) and a no-event group (203 [85.7\%] subjects) according to the occurrence of cardiovascular events.

The univariate statistical analysis demonstrated that patients in the events group manifested a reduced LVEF $(p<0.01)$, increased NYHA class $(p<0.05)$, a higher BNP plasma level on admission but also at discharge $(p<0.01)$ and impaired renal function ( $p<0.01)$ compared to subjects in the noevent group (Table I). The water composition analysis demonstrated a higher fluid overload on admission in the event group $(p<0.05)$, a difference that disappeared at discharge after diuretic therapy. No differences were found in terms of non-invasive cardiac output and cardiac index at rest or in the 6MWT (Table I). It should be underlined that $8 / 34$ patients in the events group were not able to perform the 6MWT because of confinement to bed or presenting a high degree of disability necessitating use of a wheelchair. It should be considered a limitation in the analysis of the test (see below).

Moreover, in the two groups no differences in terms of age, aetiology of CHF, or presence of atrial fibrillation emerged. The prescriptions of ACE inhibitors, angiotensin receptor blockers, and $\beta$-blockers showed a high prevalence, also considering the comorbidity (20\% suffered from hypertension and $42 \%$ suffered from ischaemic heart disease) (Table I); patients were equally treated as regards other drugs (Table I). The amount of variation regarding the in-hospital reduction of plasma BNP (delta BNP; $p=0.99$ ) or improved NYHA class (delta NYHA; $p=0.3$ ) or, finally, amelioration of fluid overload (delta resistance; $p=0.9$ ) did not dif- 
Mauro Feola, Enrico Lombardo, Marzia Testa, Enrico Avogadri, Salvatore Piccolo, Antonello Vado

Table I. Main clinical, echocardiographic and functional characteristics of patients with congestive heart failure

\begin{tabular}{|c|c|c|c|}
\hline Parameters & All $(n=237)$ & Events group $(n=34)$ & No-event group $(n=203)$ \\
\hline Age [years] & $71.1 \pm 10.1(30-89)$ & $72 \pm 7.9$ & $70.7 \pm 10.4$ \\
\hline $\operatorname{Sex}(M / F)[\%]$ & $153 / 84$ & $27 / 7$ & $124 / 79$ \\
\hline NYHA on discharge & $1.9 \pm 0.5(1-4)$ & $2.1 \pm 0.7$ & $1.94 \pm 0.4^{\star}$ \\
\hline \multicolumn{4}{|l|}{ Echocardiographic parameters } \\
\hline LV ejection fraction [\%] & $43.2 \pm 16.2(10-77)$ & $33.7 \pm 15.7$ & $44.5 \pm 15.8^{* \star}$ \\
\hline LV end systolic diameter [mm] & $46.5 \pm 23.6(15-216)$ & $85.5 \pm 57.9$ & $53.2 \pm 12.5^{\star *}$ \\
\hline LV end diastolic diameter [mm] & $57.2 \pm 25.6(24-257)$ & $119 \pm 16$ & $122 \pm 17^{\star *}$ \\
\hline Diastolic filling pattern \% (I/II/III) & $55 / 23 / 22$ & $23 / 12 / 65$ & $62 / 25 / 13$ \\
\hline BNP admission [pg/ml] & $593.4 \pm 717.7(80-5000)$ & $943.6 \pm 579.8$ & $536.4 \pm 723.2^{\star \star}$ \\
\hline BNP on discharge $[\mathrm{pg} / \mathrm{ml}]$ & $401.3 \pm 501.7(80-5000)$ & $750.2 \pm 527.3$ & $340.7 \pm 474.3^{* *}$ \\
\hline Serum creatinine $[\mathrm{mg} / \mathrm{dl}]$ & $1.3 \pm 0.8(0.5-8)$ & $1.7 \pm 0.6$ & $1.2 \pm 0.8^{\star \star}$ \\
\hline Haemoglobin [g/dl] & $12.2 \pm 1.9(7-17)$ & $12.5 \pm 1.4$ & $12.2 \pm 2$ \\
\hline Cardiac output [l/min] & $3.8 \pm 1.1(1.6-6.4)$ & $3.7 \pm 1.4$ & $3.8 \pm 0.9$ \\
\hline Cardiac index [l/min/mq] & $2.1 \pm 0.6(0.9-3.7)$ & $1.9 \pm 0.7$ & $2.1 \pm 0.5$ \\
\hline Resistance admission [ohm/mq] & $296.4 \pm 69.5(177-517)$ & $274.8 \pm 59.2$ & $303.3 \pm 71.7^{\star}$ \\
\hline Resistance on discharge [ohm/mq] & $274.6 \pm 64.1(157-425)$ & $259.5 \pm 69.5$ & $281.4 \pm 61.2$ \\
\hline Reactance admission [ohm/mq] & $28.7 \pm 8.1(11-57)$ & $28.4 \pm 9.5$ & $28.8 \pm 7.6$ \\
\hline Reactance on discharge [ohm/mq] & $27.8 \pm 7.5(13-46)$ & $28.7 \pm 7.6$ & $27.4 \pm 7.5$ \\
\hline 6-min walk test on discharge [m] & $358.5 \pm 108(80-540)$ & $347.1 \pm 82.8$ & $359.6 \pm 110.6$ \\
\hline Delta NYHA & & $1.04 \pm 0.6$ & $0.92 \pm 0.6$ \\
\hline Delta BNP [pg/ml] & & $245.1 \pm 310.8$ & $245.04 \pm 599.1$ \\
\hline Delta resistance $[\mathrm{ohm} / \mathrm{mq}]$ & & $5.2 \pm 63.3$ & $7.2 \pm 70.3$ \\
\hline \multicolumn{4}{|l|}{ Main aetiology [\%] } \\
\hline Ischaemic heart disease & 42 & 67 & 55 \\
\hline Hypertension & 20 & 10 & 13 \\
\hline Idiopathic & 14 & 9 & 10 \\
\hline Valvular heart disease & 24 & 16 & 22 \\
\hline \multicolumn{4}{|l|}{ Other comorbidities [\%] } \\
\hline Atrial fibrillation & 31 & 35 & 30 \\
\hline Diabetes mellitus & 34 & 58 & 31 \\
\hline \multicolumn{4}{|l|}{ Treatment [\%] } \\
\hline ACE inhibitors & 78 & 80 & 76 \\
\hline$\beta$-Blockers & 69 & 70 & 67 \\
\hline Angiotensin receptor blockers & 15 & 16 & 14 \\
\hline Digoxin & 55 & 63 & 52 \\
\hline Spironolactone & 57 & 53 & 59 \\
\hline Diuretics & 92 & 94 & 87 \\
\hline
\end{tabular}

The values are expressed as mean + standard deviation, unless otherwise specified.

Delta NYHA - NYHA discharge-NYHA admission, delta BNP - BNP admission-BNP discharge, delta resistance - resistance admission-resistance discharge. The ranges of values are reported in parentheses. ${ }^{*} p<0.05,{ }^{* *} p<0.01$ Pearson $\chi^{2}$

ferentiate between CHF patients who experienced cardiovascular events or not (Table I). The followup of this study lasted $408 \pm 255$ days. During the 14-month follow-up 15 patients (6.3\%) died, one
(0.4\%) underwent cardiac transplantation and 18 (7.6\%) were readmitted for CHF (events group); in $203(85.6 \%)$ no events were observed (no-events group). 
In multivariate Cox analysis only $\operatorname{LVEF}(p=0.0009)$, plasma creatinine $(p=0.006)$ and BNP at discharge $(p=0.001)$ were significantly associated with adverse mid-term outcome. Age $(p=0.6)$ and the admission value of plasma BNP $(p=0.05)$ were not significantly correlated with clinical outcome. The Kaplan-Meier survival curves demonstrated that an LVEF $<40$ or $\geq 40 \%$ and a left ventricular end-systolic diameter (LVESD) of $<47 \mathrm{~mm}$ or $\geq 47 \mathrm{~mm}$ influenced prognosis ( $p=0.04$; log rank 4.2 and $p=0.002$, log rank 9.15 respectively) (Figures 2-3) and that a stratification according to serum creatinine $(<1.5 \mathrm{mg} / \mathrm{dl}$, $1.5-2 \mathrm{mg} / \mathrm{dl}$ or $>2 \mathrm{mg} / \mathrm{dl}$ ) identified three different event-free curves ( $p=0.00001$, log rank 28.76) (Figure 4). In Figures 5 and 6 the event-free curves are depicted according to the plasma value of BNP at admission or at discharge (value $<250 \mathrm{pg} / \mathrm{ml}$ or $\geq 250 \mathrm{pg} / \mathrm{ml}$ ), showing a significant predictive val-

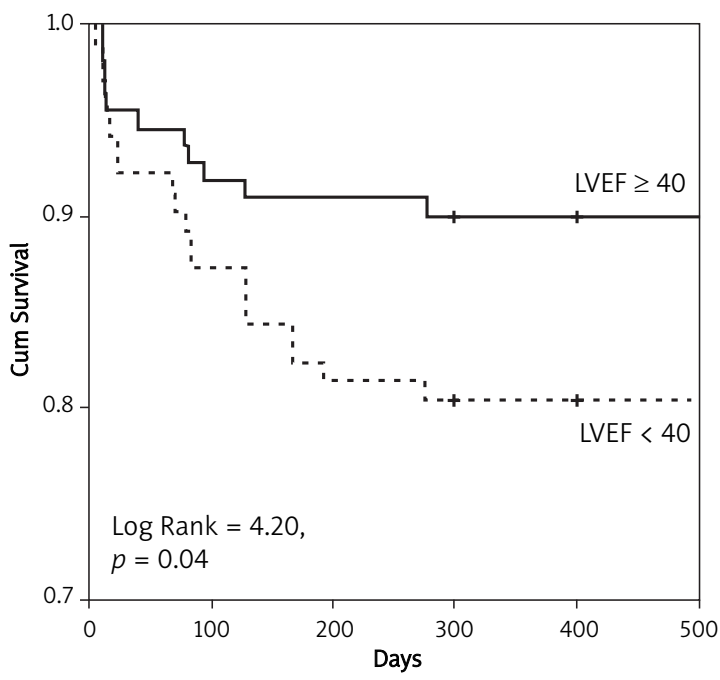

Figure 2. Event-free curves in CHF patients according to the LVEF

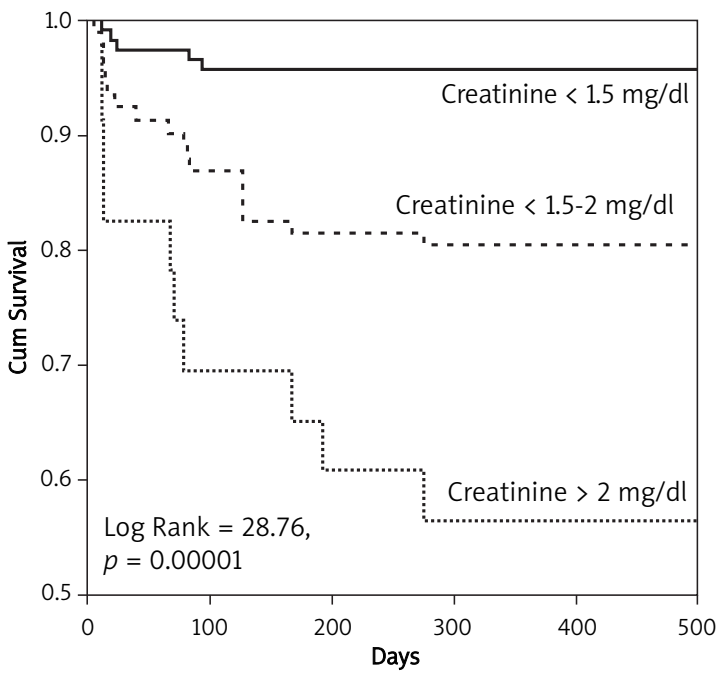

Figure 4. Event-free curves in CHF patients according to plasma creatinine ue for the discharge value ( $p=0.001$, log rank 10.7 at discharge, $p=0.05$, log rank 3.7 at admission). In Figure 7, significantly different event-free curves according to the three diastolic filling patterns (type 1-3) emerged ( $p=0.0001$, log rank 22.44). Finally, adding two parameters (creatinine < or $>1.5 \mathrm{mg} / \mathrm{dl}$ and BNP < or > $250 \mathrm{pg} / \mathrm{ml}$ ), four different survival curves were obtained ( $p=0.0001$; log rank 21.09), in which the clinical significance of renal function clearly emerged (Figure 8).

\section{Discussion}

From this study it emerged that CHF patients discharged after an acute decompensation had a severe adverse clinical outcome (death, cardiac transplantation and hospital readmission) in 14.3\% during a follow-up lasting 14 months. Elderly

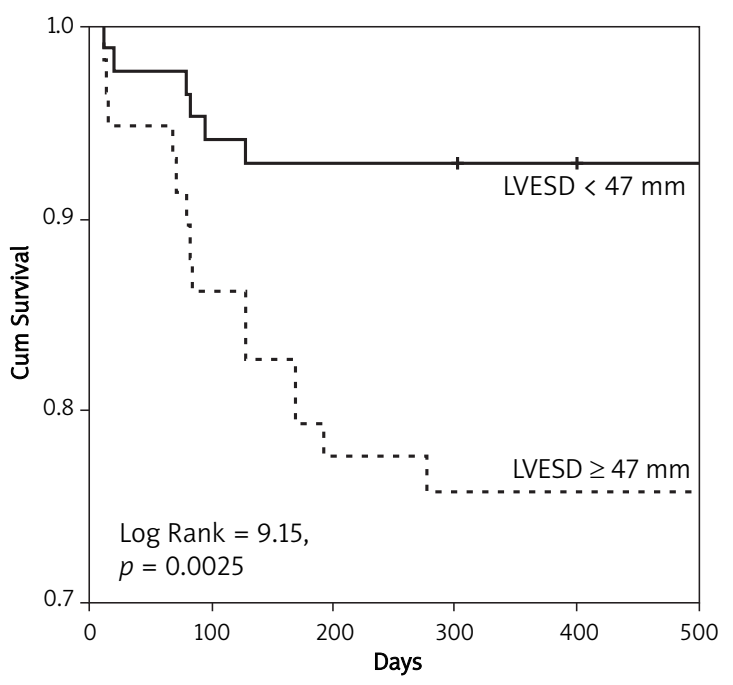

Figure 3. Event-free curves in CHF patients according to the left ventricular systolic diameter

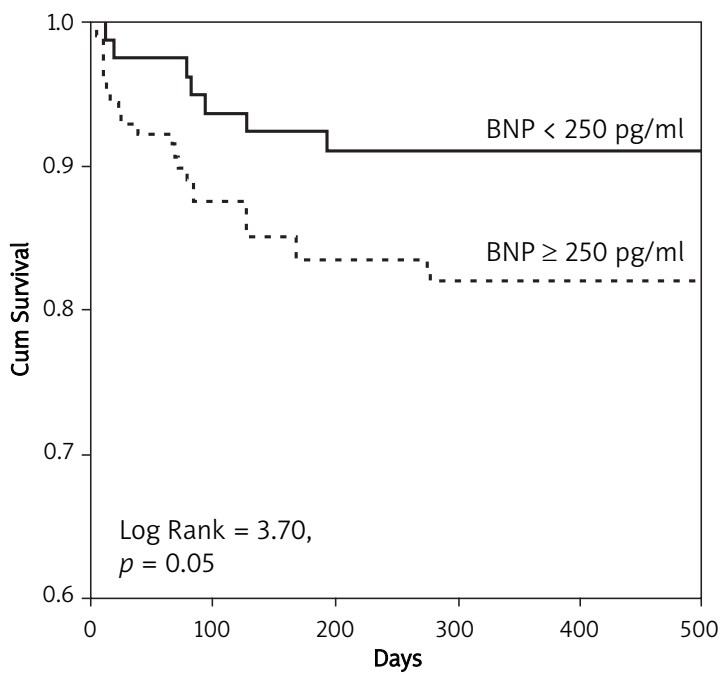

Figure 5. Event-free curves in CHF patients according to plasma BNP at admission 


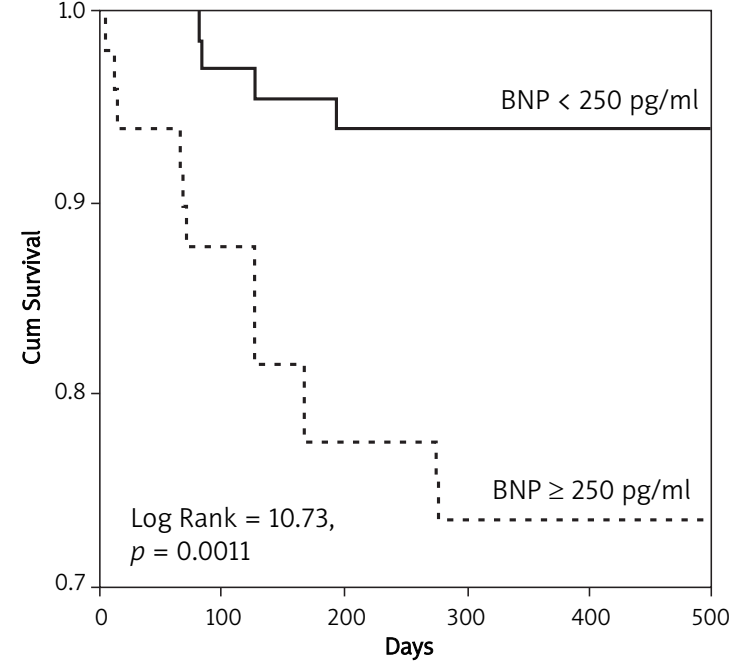

Figure 6. Event-free curves in CHF patients according to plasma BNP at discharge

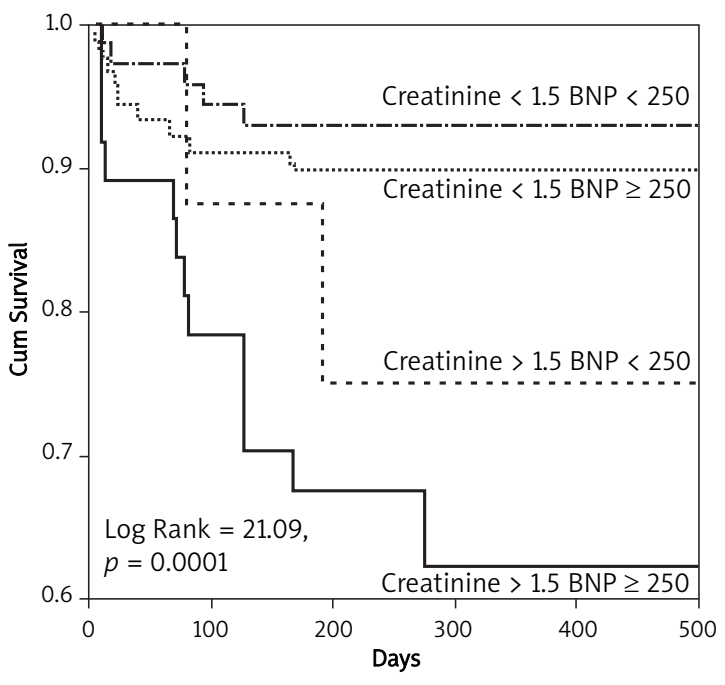

Figure 8. Event-free curves in CHF patients according to plasma BNP at discharge added to serum creatinine

patients with CHF represent most subjects (70\%) admitted to hospitals for acute cardiac decompensation; the length of hospitalization lasts usually $>2$ weeks in geriatric wards and readmission is frequent [18]. Recently, the OPTIMIZE-HF study [19], which included more than $30000 \mathrm{CHF}$ patients discharged from 215 hospitals, described a short length of hospitalization (4 days) but a $21.3 \%$ rate of readmission within 30 days. The study showed that an early (one-week) outpatient clinical followup after discharge resulted in a lower probability of being readmitted within 30 days. Considering the huge number of $\mathrm{CHF}$ patients discharged from our hospital, easy and practical prognostic parameters able to predict adverse outcomes are essential in order to allocate our resources correctly.

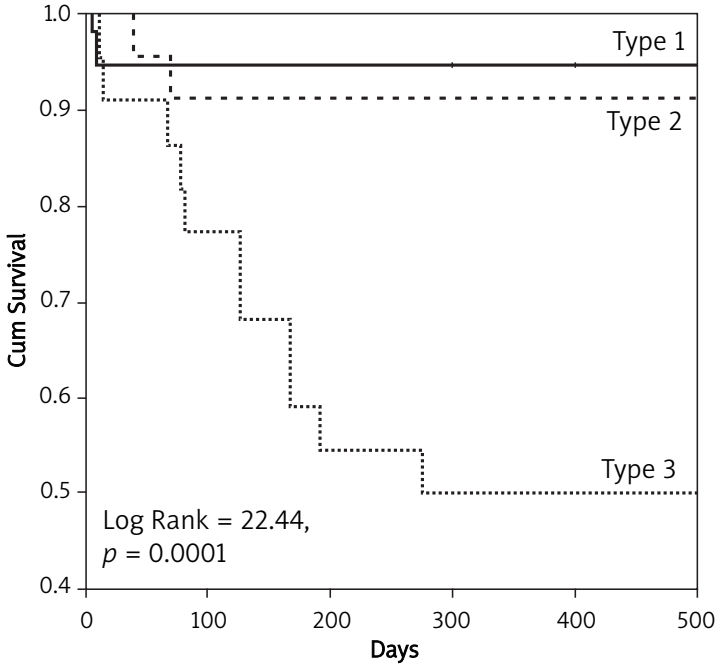

Figure 7. Event-free curves in CHF patients according to diastolic filling pattern obtained with transthoracic echocardiography before discharge

The first strong prognostic parameter in those patients was a plasma BNP value at discharge $\geq 250 \mathrm{pg} / \mathrm{ml}(p=0.001$, log rank 10.7). This confirms previous reports $[8,20]$ in which the pre-discharge values of BNP identified an adverse outcome (death or hospital re-admission) at 6-month follow-up. According to the practical approaches to treating CHF patients published by Maisel [21], there could be identified a 'wet BNP' in patients with CHF and fluid overload and a 'dry BNP' after optimization of therapy. In our population only the 'dry BNP' (at discharge) predicted cardiovascular events, because it identified the real state of neurohormonal modulation due to systolic/diastolic heart dysfunction and left ventricular enlargement. This was confirmed by the lack of significance of the amount of reduction of plasma BNP during hospitalization (delta BNP), which might be translated as: what matters is not how much the optimized therapy has reduced the BNP of your patient, but what is the value at discharge.

The second strong predictor of adverse outcome was renal dysfunction. The presence of a chronic cardio-renal syndrome, recently classified as type 2 of the cardio-renal syndromes [22], has been reported in $63 \%$ of patients admitted for $\operatorname{CHF}[23,24]$. Moreover, the ADHERE registry highlighted that the in-hospital mortality risk increased considerably in patients with urea nitrogen $\geq 43 \mathrm{mg} / \mathrm{dl}$ and plasma creatinine $\geq 2.75 \mathrm{mg} / \mathrm{dl}$ [25]. In our population serum creatinine was higher in the event group $(p<0.01)$ and alone identified (divided into three groups: $<1.5 \mathrm{mg} / \mathrm{dl}, 1.5-2 \mathrm{mg} / \mathrm{dl}$ and $>2 \mathrm{mg} / \mathrm{dl}$ ) three different cumulative survival curves (Figure 4). Moreover, adding data of renal function with discharge plasma BNP, four different cumulative survival curves emerged (BNP $<250 \mathrm{pg} / \mathrm{ml}$ and creatinine $<1.5 \mathrm{mg} / \mathrm{dl}$, BNP $<250 \mathrm{pg} / \mathrm{ml}$ and creatinine 
$>1.5 \mathrm{mg} / \mathrm{dl}$, BNP $>250 \mathrm{pg} / \mathrm{ml}$ and creatinine $<1.5 \mathrm{mg} /$ $\mathrm{dl}$, and BNP > $250 \mathrm{pg} / \mathrm{ml}$ and creatinine $>1.5 \mathrm{mg} / \mathrm{dl}$ ) (Figure 8 ), in which there clearly appeared the prognostic significance of serum creatinine $>1.5 \mathrm{mg} / \mathrm{dl}$. Therefore, this analysis identified a simple and practical combination of two markers for identifying a high-risk CHF patient at discharge for a mediumterm follow-up, which should be strictly monitored and controlled in order to avoid adverse clinical outcome. Clinicians whose take care of CHF patients might predict different clinical outcome combining two simple and inexpensive parameters (plasma BNP and plasma creatinine).

Finally, this study confirmed the prognostic significance of simple echocardiographic parameters, such as LVEF, left ventricular end-systolic diameter (LVESD) and the diastolic filling pattern. In the ESC Guidelines on chronic heart failure [26], the left ventricle was defined as enlarged when the endsystolic diameter was $>45 \mathrm{~mm}$. In patients who survived a myocardial infarction [27] and in cardiomyopathies [28], LV diameters obtained with $M$ mode had strong value for predicting cardiovascular death in long-term follow-up. In our population, the cut-off of $47 \mathrm{~mm}$ for LVESD guaranteed a significant powerful stratification for cardiovascular events. The prognostic role of mitral pattern inflow in CHF subjects was highlighted in the metaanalysis of Whalley et al. [29], in which a mortality rate of $22.7 \%$ and an odds ratio for all-cause mortality of 4.36 emerged (average follow-up between 3 months and 5 years). In CHF patients with idiopathic dilated cardiomyopathy, the presence of a restrictive filling pattern was associated with a sixfold (OR 6.65) increase in mortality. The mortality rate in post-MI patients with a restrictive pattern in the echocardiogram was $31.8 \%$ (average followup between 2 weeks and 5 years) with a calculated odds ratio of 4.10 [30]. The presence of a predischarge restrictive filling pattern in CHF patients after an acute episode of cardiac decompensation corresponded to a high short-term mortality (14\%) and readmission rate (42.4\%) [31]. Data obtained in this study underlined the importance of the echocardiographic assessment of diastolic filling pattern in CHF patients, able to differentiate prognosis in type 3 vs. the non-restrictive pattern ( $p=0.0001$; log-rank 22.44) and providing robust prognostic information. Finally, this experience failed to demonstrate clinical predictive value of non-invasive determination of CO or SV (Definition missing) at rest, that might be tested during exercise in order to discriminate exercise limitation due to left ventricle pump failure [32].

In conclusion, in CHF patients discharged with bioimpedance evidence of normal hydration after an episode of acute decompensation, plasma BNP $\geq 250 \mathrm{pg} / \mathrm{ml}$ and mild renal impairment seemed to be the strongest predictor of mid-term adverse outcome. Simple echocardiographic parameters (LVEF, LVESD and diastolic filling pattern) might help in determining clinical outcome.

In the interpretation of the 6MWT results the complete inability of $23.5 \%$ of patients in the events group to perform the test might be underestimated. In fact, Passantino et al. [33] demonstrated that the increase in walking distance was significantly associated with survival in CHF patients who walked $<340 \mathrm{~m}$ at baseline, confirming the highly prognostic value of $6 \mathrm{MWT}$ in those patients.

\section{References}

1. Swedberg K, Cleland J, Dargie H, et al. Guidilines for the diagnosis and treatment of chronic heart failure: executive summary (update 2005): the Task Force for the Diagnosis and Treatment of chronic Heart Failure of the European Society of Cardiology. Eur Heart J 2005; 26: 1115-40.

2. Fonarow GC, Adams KF, Abraham WT, Yancy CW, Boscardin WJ; ADHERE Scientific Advisory Committee. Risk stratification for in-hospital mortality in acutely decompensated heart failure. JAMA 2005; 293: 572-80.

3. Boland DG, Abraham WT. Natriuretic peptides in heart failure. Congestive Heart Fail 1998; 4: 23-33.

4. McDonagh TA, Robb SD, Murdoch DR, et al. Biochemical detection of left-ventricular systolic dysfunction. Lancet 1998; 351: 9-13.

5. Maisel AS, Krishnaswamy P, Nowak RM, et al. Rapid measurement of B-type natriuretic peptide in the emergency diagnosis of heart failure. N Engl J Med 2002; 347: 161-7.

6. Berger R, Huelsman M, Strecker K, et al. B-type natriuretic peptide predicts sudden death in patients with chronic heart failure. Circulation 2002; 105: 2392-7.

7. Latini R, Masson S, Anand I, et al.; Val-HeFT Investigators. The comparative prognostic value of plasma neurohormones at baseline in patients with heart failure enrolled in Val-HeFT. Eur Heart J 2004; 25: 292-9.

8. Feola M, Aspromonte N, Canali C, et al. Prognostic value of plasma brain natriuretic peptide, urea nitrogen and creatinine in outpatients > 70 years of age with heart failure. Am J Cardiol 2005; 96: 705-9.

9. Wang TJ, Larson MG, Levy D, et al. Plasma natriuretic peptide levels and the risk of cardiovascular events and death. N Engl J Med 2004; 350: 655-63.

10. McKee PA, Castelli WP, McNamara PM, Kannel WB. The natural history of CHF. The Framingham study. N Engl J Med 1971; 285: 1442-6.

11. Peacock WF. Rapid optimization: strategies for optimal care of decompensated congestive heart failure patients in the emergency department. Rev Cardiovasc Med 2003; 3: S41-8.

12. Sahn DJ, Demaria A, Kisslo J, Weyman A. The committee on $\mathrm{M}$ mode standardisation of the American Society of Echocardiography: recommendations regarding quantification in M-mode echocardiography: results of a survey of echocardiographic measurements. Circulation 1978; 58: 1072-83.

13. Garcia MJ, Thomas JD, Klein AL. New Doppler echocardiographic applications for the study of diastolic function. J Am Coll Cardiol 1998; 32: 865-75.

14. Agostoni PG, Cattadori G, Apostolo A, et al. Non-invasive measurement of cardiac output during exercise by inert 
gas rebreathing technique: a new tool for heart failure evolution. J Am Coll Cardiol 2005; 46: 1779-83.

15. ATS Statement: guidelines for the six-minute walk test. ATS Committee on Proficiency Standards for Clinical Pulmonary Function Laboratories. Am J Respir Crit Care Med 2002; 166: 111-7.

16. Bioelectrical impedance analysis in body composition measurement. Proceeding of a National Institutes of Health Technology Assessment Conference. Bethesda, Maryland, December 12-14, 1994. Am J Clin Nutr 1996; 64 Suppl: 387S-532S.

17. Piccoli A. Patterns of bioelectrical impedance vector analysis: learning from electrocadiography and forgetting electric circuit models. Nutrition 2002; 18: 520-1.

18. Di Lenarda A, Scherillo M, Maggioni AP, et al. Current presentation and management of heart failure in cardiology and internal medicine hospital units: a tale of words. The TEMISTOCLE study. Am Heart J 2003; 146: E12.

19. Hernandez AF, Greiner MA, Fonarow GC, et al. Relationship between early physician follow-up and 30-day readmission among Medicare beneficiaries hospitalized for heart failure. JAMA 2010; 303: 1716-22.

20. Logeart D, Thabut G, Jourdain P, et al. Predischarge B-type natriuretic peptide assay for identifying patients at high risk of re-admission after decompensated heart failure. J Am Coll Cardiol 2004; 43: 635-41.

21. Maisel AS. Practical approaches to treating patients with acute decompensated heart failure. J Card Fail 2001; 7 (Suppl 1): 13-7.

22. RoncoC, McCullogh P, Anker SD, et al. Cardio-renal syndromes: report from the consensus Conference of the Acute Dialysis Quality Initiative. Eur Heart J 2010; 6: 703-11.

23. National Kidney Foundation. K/DOQI clinical practice guidelines for chronic kidney disease: evaluation, classification and stratification. Am J Kidney Dis 2002; 39 (Suppl 2): S1-266.

24. Heywood JT, Fonarow GC, Costanzo MR, Mathur VS, Wignesrawan JR, Wynne J. High prevalence of renal dysfunction and its impact on outcome in 118,465 patients hospitalized with acute decompensated heart failure: a report from the ADHERE database. J Card Fail 2007; 13: 422-30.

25. Fonarow GC, Adams KF, Abraham WT, Yancy CW, Boscardin WJ; ADHERE Scientific Advisory Commettee. Risk stratification for in-hospital mortality in acutely decompensated heart failure. Classification and regression tree analysis. JAMA 2005; 293: 572-80.

26. Dickstein K, Cohen-Solal A, Filippatos G, et al. ESC Guidelines for the diagnosis and treatment of acute and chronic heart failure 2008. Eur Heart J 2008; 29: 2388-442.

27. Eriksson SV, Caidahl K, Hamsten A, de Faire U, Rehnquist N, Lindvall K. Long-term prognostic significance of $M$ mode echocardiography in young men after myocardial infarction. Br Heart J 1995; 74: 124-30.

28. Sachero A, Casazza F, Recalcati F, et al. Clinical and prognostic significance of echocardiographic parameters in dilated cardiomyopathy: a prospective study on 255 patients. The Italian Multicenter Study of Cardiomyopathies Group. G Ital Cardiol 1992; 22: 1077-90.

29. Whalley GA, Gamble G, Doughty R. The prognostic significance of restrictive filling associate with heart failure: a meta-analysis. Int J Cardiol 2007; 116: 70-7.

30. Whalley GA, Gamble G, Doughty R. Restrictive diastolic filling predicts death post acute myocardial infarction: a meta-analysis. Heart 2006; 92: 1588-94.

31. Feola M, Aspromonte N, Milani L, et al. Plasma brain natriuretic peptide predicts short-term clinical outcome in heart failure patients with restrictive filling pattern. J Card Fail 2008; 14: 420-5.

32. Cattadori G, Salvioni E, Gondoni E, Agostoni PG. Evaluation of noninvasive exercise cardiac output determination in chronic heart failure pateints: a proposal of a new diagnostic and prognostic method. J Cardiovasc Med 2010; 12: 19-27.

33. Passantino A, Lagioia R, Mastropasqua F, Scrutinio D. Short-term change in distance walked in $6 \mathrm{~min}$ is an indicator of outcome in patients with chronic heart failure in clinical practice. J Am Coll Cardiol 2006; 48: 99-105. 